Subscriber access provided by King Abdullah University of Science and Technology Library

Letter

\title{
Direct observation of current-induced motion of \\ a 3D vortex domain wall in cylindrical nanowires
}

Yurii P. Ivanov, Andrey Chuvilin, Sergei Lopatin, Hanan Mohammed, and Jurgen Kosel

ACS Appl. Mater. Interfaces, Just Accepted Manuscript • Publication Date (Web): 08 May 2017

Downloaded from http://pubs.acs.org on May 15, 2017

\section{Just Accepted}

"Just Accepted" manuscripts have been peer-reviewed and accepted for publication. They are posted online prior to technical editing, formatting for publication and author proofing. The American Chemical Society provides "Just Accepted" as a free service to the research community to expedite the dissemination of scientific material as soon as possible after acceptance. "Just Accepted" manuscripts appear in full in PDF format accompanied by an HTML abstract. "Just Accepted" manuscripts have been fully peer reviewed, but should not be considered the official version of record. They are accessible to all readers and citable by the Digital Object Identifier (DOI®). "Just Accepted" is an optional service offered to authors. Therefore, the "Just Accepted" Web site may not include all articles that will be published in the journal. After a manuscript is technically edited and formatted, it will be removed from the "Just Accepted" Web site and published as an ASAP article. Note that technical editing may introduce minor changes to the manuscript text and/or graphics which could affect content, and all legal disclaimers and ethical guidelines that apply to the journal pertain. ACS cannot be held responsible for errors or consequences arising from the use of information contained in these "Just Accepted" manuscripts. 


\title{
Direct Observation of Current-Induced Motion of a
}

\section{D Vortex Domain Wall in Cylindrical Nanowires}

\author{
Yurii P. Ivanov ${ }^{\dagger,+, \xi, *}$, Andrey Chuvilin ${ }^{\|, \perp}$, Sergey Lopatin ${ }^{\dagger}$, Hanan Mohammed ${ }^{\dagger}$, Jurgen Kosel ${ }^{\dagger}$ \\ ${ }^{\dagger}$ King Abdullah University of Science and Technology, Thuwal, 23955-6900, Saudi Arabia \\ ${ }^{\star}$ Erich Schmid Institute of Materials Science, Austrian Academy of Sciences, Jahnstrasse 12, A- \\ 8700, Leoben, Austria \\ $\S$ School of Natural Sciences, Far Eastern Federal University, 690950, Vladivostok, Russia \\ " CIC nanoGUNE Consolider, Av. de Tolosa 76, 20018, San Sebastian, Spain \\ ${ }^{\perp}$ IKERBASQUE, Basque Foundation for Science, Maria Diaz de Haro 3, 48013, Bilbao, Spain
}

KEYWORDS: current-driven domain wall motion, nanowires, assembled nanostructures, in-situ electron microscopy, 3D memory.

\begin{abstract}
The current-induced dynamics of 3D magnetic vortex domain walls in cylindrical $\mathrm{Co} / \mathrm{Ni}$ nanowires are revealed experimentally using Lorentz microscopy and theoretically using micromagnetic simulations. We demonstrate that a spin-polarized electric current can control the reversible motion of 3D vortex domain walls, which travel with a velocity of a few hundred
\end{abstract}


meters per second. This finding is a key step in establishing fast, high-density memory devices based on vertical arrays of cylindrical magnetic nanowires.

Modern information storage technology is advancing toward the realization of $3 \mathrm{D}$ devices. ${ }^{1-4} \mathrm{~A}$ very promising concept for 3D magnetic memory devices called vertical race-track memory was proposed in $2008,{ }^{5}$ in which current-driven domain wall (DW) motion in magnetic nanowires is utilized to manipulate information. Magnetic domains, which are separated by DWs, represent bits of information, and the DW position and motion (i.e. information storage and transfer) can be controlled by spin-polarized electric currents.

The main challenge in realizing a race-track memory with cylindrical nanowires is posed by the current standard microfabrication methods. For example, one way to increase memory density is by stacking multiple storage layers in a single device. However, even a small number of layers significantly complicates the fabrication process. ${ }^{2}$ The standard methods are highly effective at fabricating planar devices, but are very limited when it comes to out-of-plane structures. Hence, there is a need for more efficient fabrication methods.

Recently, we proposed an information storage medium composed of dense arrays of cylindrical multisegmented $\mathrm{Co} / \mathrm{Ni}$ nanowires arranged vertically on a substrate and fabricated using a simple electrodeposition technique. ${ }^{10}$ Cylindrical magnetic nanowires can be fabricated efficiently inside nanoporous templates and display various unique properties. ${ }^{6-9} \mathrm{We}$ demonstrated that the interfaces between the Co and Ni segments create a periodic energy landscape and act as pinning sites for DW propagation. The DW magnetic structure is a type of $3 \mathrm{D}$ vortex ${ }^{11}$ with a Bloch point at its center. We used differential phase contrast imaging and micromagnetic simulations to study the detailed structure of a 3D vortex DW in a cylindrical 
$\mathrm{Co} / \mathrm{Ni}$ nanowire. ${ }^{10}$ The next step is to deliberately move the DWs from one pinning site to another.

The DW motion driven by spin-polarized electric currents has been thoroughly studied in planar magnetic nanowires ${ }^{12-20}$ and the intrinsic limitations of such systems are well-understood. These limitations include the Walker breakdown phenomenon, which limits the propagation speed of DWs (a crucial parameter for fast memory storage media), and unwanted pinning of DWs at imperfections in planar structures. ${ }^{12,17}$

In non-planar (cylindrical) nanowires, the probability of DWs pinning at random structural imperfections significantly decreases, and theoretical models show that 3D vortex DWs do not suffer from the Walker breakdown phenomenon. ${ }^{21-22}$ However, current-driven DW motion in cylindrical nanowires has not yet been experimentally observed.

In this work, we report the direct observation of current-driven motion of 3D vortex DWs in cylindrical Co/Ni nanowires using Lorentz - Transmission Electron Microscopy (L-TEM) with in-situ magnetic and electrical biasing. From these observations, we derive the de-pinning current density and compare it with the micromagnetic simulation results. These results suggest an exceptionally high DW velocity in cylindrical nanowires.

Multisegmented cylindrical $\mathrm{Co} / \mathrm{Ni}$ nanowires were fabricated by electrodeposition inside hexagonally ordered Aluminum Oxide Templates (AOT). The preparation details are reported elsewhere. ${ }^{10}$ Briefly, the nanowire was $80 \mathrm{~nm}$ in diameter and $21 \mu \mathrm{m}$ in length, composed of 30 alternating $\mathrm{Co}$ and $\mathrm{Ni}$ segments averaging $700 \mathrm{~nm}$ each in length. In order to access the individual nanowires for the in-situ TEM measurements, the AOT was dissolved by chemical etching. The nanowires were dispersed in ethanol and sedimented onto a transparent silicon nitride membrane on the TEM microchips (Protochip AEW) with pre-patterned Pt contacts. The 
orientation of the nanowires with respect to the Pt contacts was controlled by applying an external magnetic field. The electrical connectors between the individual nanowires and the prepatterned Pt contacts were fabricated using Focus Ion Beam (FIB)-assisted Pt deposition at low ion currents (see Supporting Information for details). Figure 1a shows a Scanning Transmission Electron Microscopy (STEM) image of the fabricated device. The Pt contacts are visible at both ends of the wire, and the pre-patterned Pt electrode appears at the top-right corner of the image. Figure 1b shows an Electron Energy Loss Spectroscopy (EELS) elemental map of the wire, revealing the sequence of Co and Ni segments. Previously, we have demonstrated that such a "bar code" structure creates pinning sites at the segments' interfaces, and that the DWs can be moved between these pinning sites by applying an external magnetic field. ${ }^{10,23}$

For the in-situ experiments, the TEM was operated in Lorentz mode with a nullified Objective Lens (OL) current to achieve field-free imaging conditions. Lorentz microscopy can easily observe the entire nanowire with a field of view that exceeds $20 \mu \mathrm{m}$, which is not achievable with the TEM holography method. L-TEM is also significantly faster (0.05-0.1 sec per frame) than, for example, the VBF-DPC method ${ }^{23}$ (20-50 sec per frame), and is not influenced by charging of the supporting SiN membrane, unlike TEM holography. The magnetization of the wire was captured by Fresnel imaging. ${ }^{24}$ Figure 1c shows the overfocused image of the nanowire at remanence, after being saturated with a magnetic field from right to left (L state). The Fresnel contrast, which appears as a white line inside the nanowire, is shifted downwards with respect to the axis of the wire. If the magnetization direction of the nanowire is reversed by saturating it with a field from left to right, (the R state) the white line appears shifted upwards (Fig. 1g). Thus, the position of the white line along the wire unambiguously indicates the direction of magnetization. Figure 1e shows the case of two domains: the L state is observed on the left side 
of the nanowire and the R state on the right side, with a DW pinned (see discussion below) between the two states (the initial DW state). Figure $1 \mathrm{~d}$ and $1 \mathrm{f}$ show two variations of the DW state, the "DW state - L state" and the "DW state - R state," respectively, emphasizing the shift in the Fresnel contrast and confirming the existence of two magnetic domains.

The magnetic state of the nanowire was manipulated by inclining the sample at 30 degrees relative to the optical axis (the direction of the magnetic field of the excited OL) and exciting the OL to a defined magnitude. This approach allows not only the reversal of the magnetization in the nanowire, but also the control of the nucleation and motion of the DWs (see Supporting Movie for an example of the periodic DW motion between the two pinning sites). The value of the external magnetic field needed to reproduce a monodomain state was 360 Oe. A smaller field of $298 \mathrm{Oe}$, henceforth referred to as the nucleation field $H_{n}$, was required for DW nucleation at the right end of the wire and DW propagation to the pinning site in the middle of the wire. The DW then remained stable until the field increased to 360 Oe, which can be referred to as the depinning field $H_{d e p}$. DW pinning and de-pinning was also confirmed by magnetoresistance measurements conducted with a separate setup (see Supporting Information). The pinning of the DW to a specific location was reliably reproduced multiple times, leading to the DW state shown in Fig. 1e. This DW state is the initial state for all current-driven DW experiments described below. 


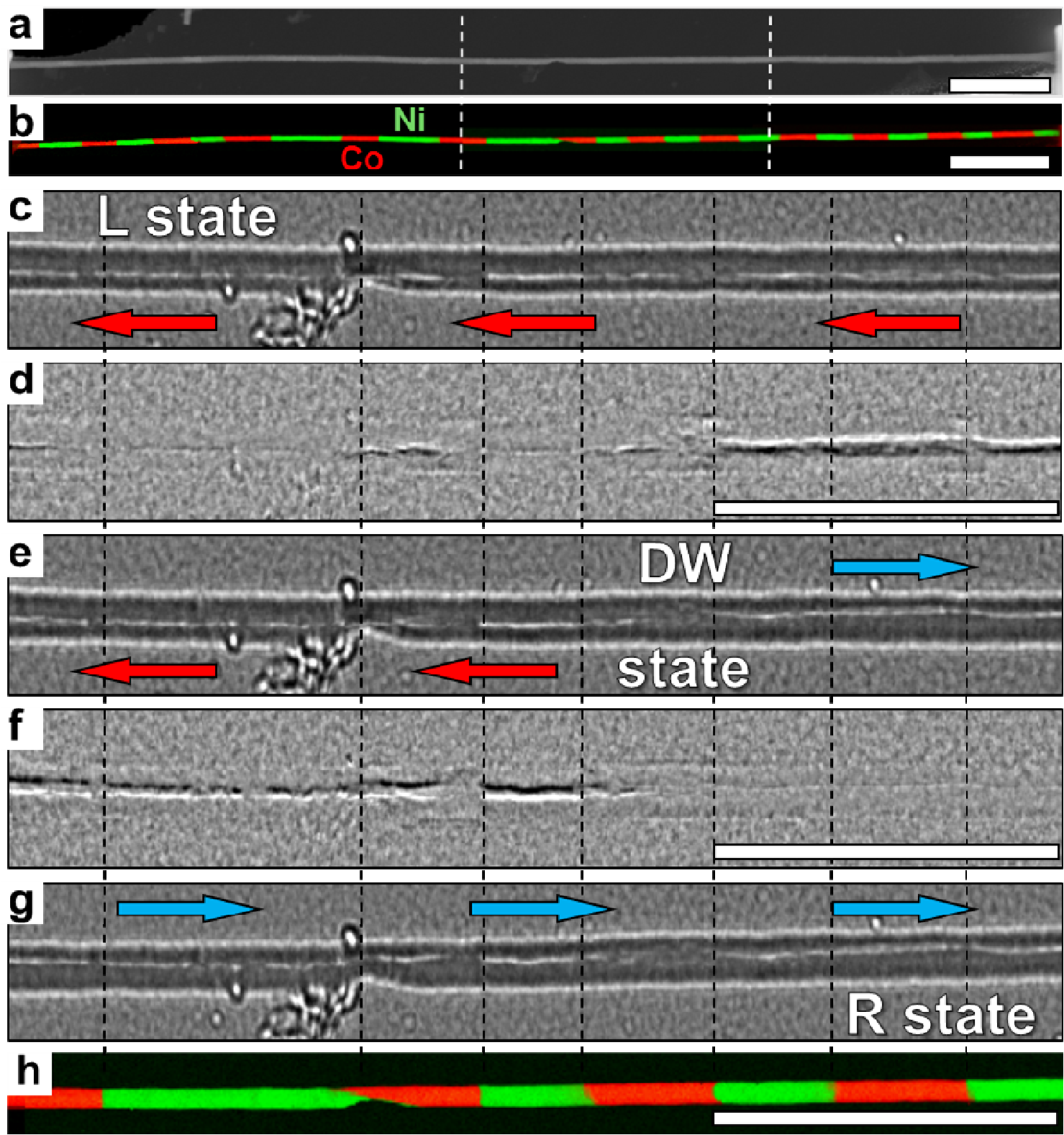

Figure 1. (a) STEM image of the device, which consists of a multisegmented $\mathrm{Co} / \mathrm{Ni}$ nanowire connected to Pt electrodes. (b) EELS elemental mapping of the multisegmented Co/Ni nanowire. (c-g) Overfocused Lorentz images (scaled to $300 \%$ in the vertical direction) of the central section of nanowire designated by white dashed lines in $\mathbf{a}$ and $\mathbf{b}$ : $\mathbf{c}$ and $\mathbf{g}$ are the $\mathrm{L}$ state and the R state, $\mathbf{e}$ is the initial state with the DW pinned at the "interface - DW state," $\mathbf{d}$ and $\mathbf{f}$ are images of the "DW state - L state," and the "DW state - R state," respectively. (h) EELS elemental mapping of 
the Co and Ni nanowire segments, the interfaces of which are designated by dashed black lines in c-g. Scale bar is $2 \mu \mathrm{m}$. The complete Lorentz images are in the Supporting Information.

Current-driven DW motion in planar nanowires typically requires electric current densities on the order of $10^{12} \mathrm{~A} / \mathrm{m}^{2}$ and nano or picosecond pulse durations. ${ }^{12}$ Our actual in-situ setup is limited to DC currents only, which ultimately restricts the applicable current magnitude: at a $10^{12}$ $\mathrm{A} / \mathrm{m}^{2} \mathrm{DC}$ current, the nanowire would warm up to over $1900 \mathrm{~K}$. We have found that the maximum current density applicable in our setup is $J_{e}=0.7 \times 10^{11} \mathrm{~A} / \mathrm{m}^{2}$. At this current density, the temperature increase is within $50 \mathrm{~K}$ (see Supporting Information for more discussion and experimental measurements). Since DW motion could not be induced at this current density, we have applied an auxiliary magnetic field of 315 Oe along the wire. This field was substantially smaller than $H_{d e p}$, so the DW remained in a stable position at the pinning site when under the action of only this field.

The application of an external magnetic field makes one of the two different magnetization directions in the domains energetically more favorable. By applying electric current in the appropriate direction, the unfavorable magnetization direction can be reversed, provided that the DW is de-pinned. In the DW configuration shown in Fig. 1e, the direction of the current should coincide with the direction of the magnetic field (i.e. the electron flow is opposite to the magnetic field). Figure 2 summarizes the experimental observations of DW de-pinning with different combinations of magnetic field and electrical current. With a suitable combination, the critical current density $J_{c}=0.6 \times 10^{11} \mathrm{~A} / \mathrm{m}^{2}$ was sufficient to de-pin the DW and transfer the nanowire to a single domain state. If the current was applied in the direction opposite to the magnetic field, the DW remained stable, even at a current density of $0.7 \times 10^{11} \mathrm{~A} / \mathrm{m}^{2}$. This 
observation eliminates the possibility that Joule heating causes the DW to de-pin (see more discussion about Joule heating in the Supporting Information).

The above measurements were repeated 10 times for the nanowire shown in Fig. 1 and for similar devices, reproducing identical results.

Initial state
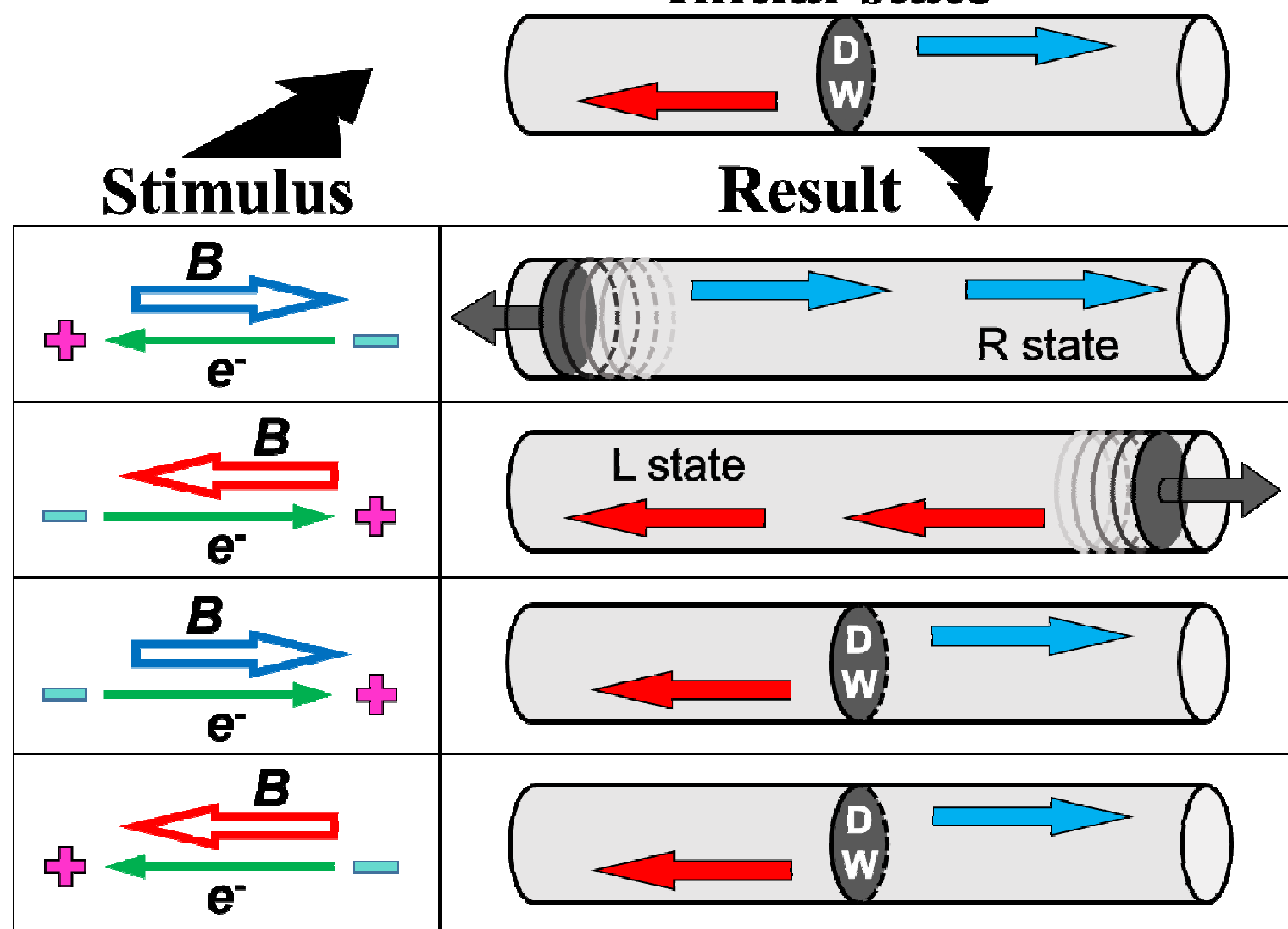

Figure 2. Diagram representing the magnetic state of the nanowire shown in Fig. 1, under external magnetic field (B) and electric current.

To get further insight into the phenomena observed by in-situ TEM measurements, we performed micromagnetic simulations of a segmented $\mathrm{Co} / \mathrm{Ni}$ nanowire. The magnetization process and DW dynamics were simulated using the OOMMF package. ${ }^{25}$ The parameters used for the simulations have been reported elsewhere. ${ }^{26}$ The simulation of the magnetic reversal 
process demonstrates a two-step reversal: (1) nucleation of the DW at the end of the Co segment at $H_{n}=240 \mathrm{Oe}$ and DW pinning at the Co/Ni interface, and (2) de-pinning at $H_{d e p}=320 \mathrm{Oe}$, which is in good agreement with the experimentally measured parameters.
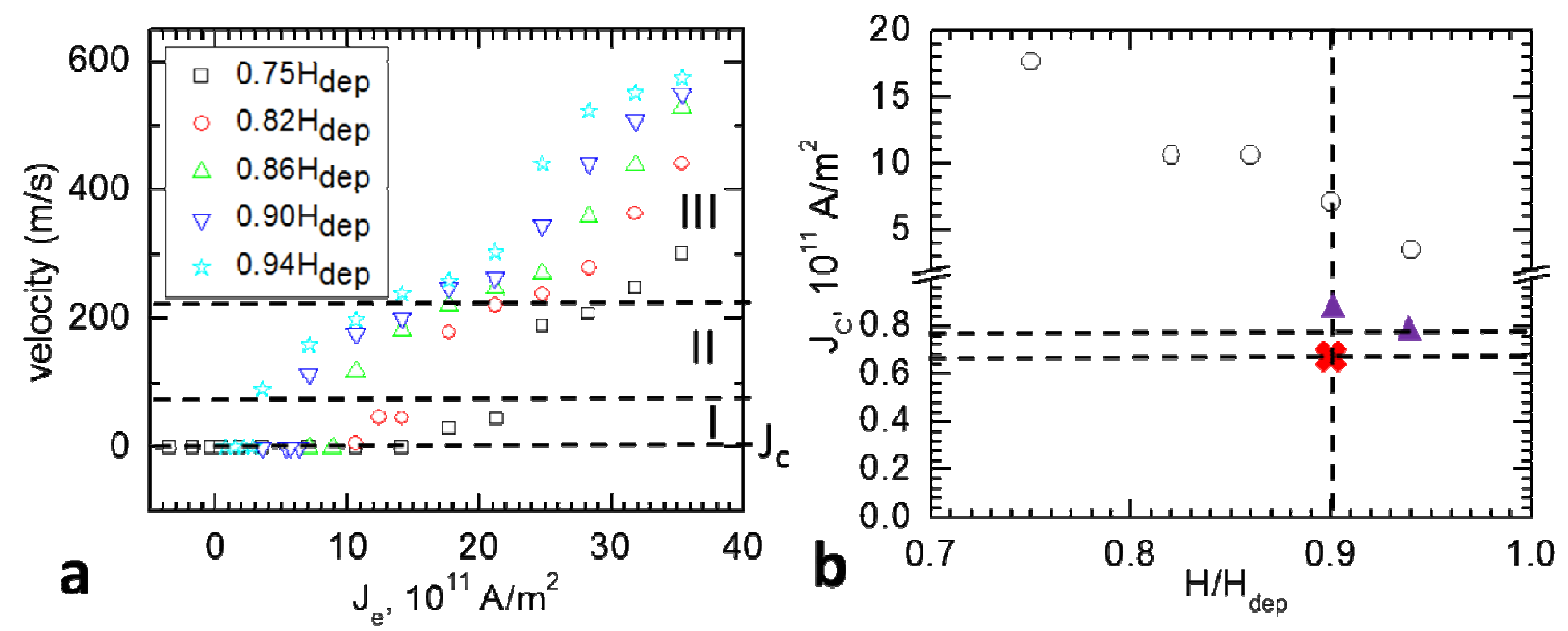

Figure 3. (a) Domain wall velocity under different current densities $J_{e}$ for various external magnetic field values with respect to the de-pinning field $H_{d e p}$, simulated at $0 \mathrm{~K}$ temperature. (b) Simulated dependence of the critical current density $J_{c}$ on the external magnetic field values at 0 $\mathrm{K}$ (circles) and $327 \mathrm{~K}$ (triangles), and experimental data at $327 \mathrm{~K}$ (red "X”).

As can be seen in Fig. 3a, there is a critical value of $J_{c}$ above which current-driven de-pinning occurs, and this value depends on the applied field. The maximum value found for the speed of the 3D DW was $500 \mathrm{~m} / \mathrm{s}$. The simulations show that the critical density of the spin-polarized current at $0 \mathrm{~K}$ decreases almost linearly when increasing the external magnetic field (Fig. 3b). Note that the simulated value of $J_{c}$ at $0 \mathrm{~K}$ is more than one order of magnitude larger than the experimental value. Therefore, we performed a micromagnetic simulation using extensions of the OOMMF code, which allow the simultaneous simulation of spin-transfer torque and thermal fluctuation effects. The $J_{c}$ value calculated for $327 \mathrm{~K}$ is shown as a triangle in Fig. 3b, and is 
very close to the one obtained experimentally (red " $\mathrm{X}$ " in Fig. 3b). There are probably additional contributions to the current-driven 3D DW motion which were not taken into account in the micromagnetic model used. Additional experiments with nanosecond current pulses and advanced ultra-fast imaging techniques ${ }^{12}$ are necessary to discover these unknown influences. The estimated current density is $2 \times 10^{11} \mathrm{~A} / \mathrm{m}^{2}$ for zero-field current-driven domain wall motion in barcode cylindrical nanowires.

Until now, difficulties in achieving reliable pinning sites and measurement complexities have prevented the demonstration of current-driven DW motion in cylindrical nanowires. In this paper, we have confirmed experimental demonstrations of current-driven DW motion in cylindrical nanowires using L-TEM. With multisegmented $\mathrm{Co} / \mathrm{Ni}$ nanowires, we reliably moved a 3D vortex DW between two pinning sites. We have thus established the parameters by which spin-polarized electric current can drive the motion of 3D DWs in such nanowires. We found that, in combination with an external magnetic field 0.9 times the field required to de-pin the DWs, the critical current density needed for DW motion is $6 \times 10^{10} \mathrm{~A} / \mathrm{m}^{2}$. In addition to the LTEM measurements, we used the OOMMF package to perform micromagnetic simulations, which are in good agreement with the experimental observations. The simulations predict a linearly decreasing value of the critical current density on the applied field and a very high speed of the DW motion. These findings, combined with the 3D nature of the nanowire, make the multisegmented $\mathrm{Co} / \mathrm{Ni}$ nanowire array a very promising candidate for a fast $3 \mathrm{D}$ data storage device. 


\section{ASSOCIATED CONTENT}

Supporting Information. This material is available free of charge on the ACS publication website. We provide methods, additional figures and information about the device setup of the in-situ TEM study, online data, and ex-situ magnetoresistance measurements (PDF). The Supporting Movies demonstrate the field-driven domain wall motion and a typical example of the micromagnetic simulation of the current-driven domain wall motion (AVI).

\section{AUTHOR INFORMATION}

\section{Corresponding Author}

* Correspondence and requests for materials should be addressed to Yurii P. Ivanov, e-mail: ivanov.yup@gmail.com

\section{Author Contributions}

Y. I. conceived the project, designed and performed in-situ TEM experiments, performed data analysis, and wrote the manuscript; A.C. and S.L. performed TEM experiments and data analysis, and contributed to the manuscript writing; J.K. supported the experiments and contributed to the manuscript writing. H.M. performed ex-situ magnetoresistance measurements and contributed to manuscript writing.

\section{Notes}

The authors declare no competing financial interests.

\section{ACKNOWLEDGMENT}

Research reported in this publication was supported by the King Abdullah University of Science and Technology (KAUST). 


\section{REFERENCES}

1. IBM News Room Page. http://www-03.ibm.com/press/us/en/pressrelease/36125.wss (accessed Dec 1, 2011).

2. Hwang, C. S. Prospective of Semiconductor Memory Devices: from Memory System to Materials. Adv. Electron. Mater. 2015, 1, 1400056.

3. Song, S; Cho, B.; Kim, T.-W.; Ji, Y.; Jo, M.; Wang, G.; Choe, M.; Kahng, Y.H.; Hwang, H.; Lee, T. Three-Dimensional Integration of Organic Resistive Memory Devices. Adv. Mater. 2010, 22, 5048-5052.

4. Prince, B. Vertical 3D Memory Technologies, 1st ed.; John Wiley \& Sons: Chichester, $\mathrm{UK}, 2014$.

5. Parkin, S. S. P.; Hayashi, M.; Thomas, L. Magnetic Domain-Wall Racetrack Memory. Science 2008, 320 (5873), 190-194.

6. Ivanov, Yu. P.; Alfadhel, A.; Alnassar, M.; Perez, J.; Vázquez, M.; Chuvilin, A.; Kosel, J. Tunable Magnetic Nanowires for Biomedical and Harsh Environment Applications. Sci. Rep. 2016, 6, 24189.

7. Ivanov, Yu. P.; Chuvilin, A.; Vivas, L.G.; Kosel, J.; Chubykalo-Fesenko, O.; Vázquez, M. Single Crystalline Cylindrical Co Nanowires - Towards Dense Magnetic Vortex Array. Sci. Rep. 2016, 6, 23844.

8. Vilanova, V. E.; Ivanov, Y. P.; Mohammed, H.; Kosel, J. A Detailed Study of Magnetization Reversal in Individual Ni Nanowires. Appl. Phys. Lett. 2015, 106, 032403. 
9. Ivanov, Yu. P.; Trabada, D. G.; Chuvilin, A.; Kosel, J.; Chubykalo-Fesenko, O.;

Vázquez, M. Crystallographically Driven Magnetic Behaviour of Arrays of Monocrystalline Co Nanowires. Nanotechnology 2014, 25, 475702.

10. Ivanov, Yu. P.; Chuvilin, A.; Lopatin, S.; Kosel, J. Modulated Magnetic Nanowires for Controlling domain Wall Motion: Towards 3D Magnetic Memories. ACS Nano 2016, 10, 53265332.

11. Wang, J.; Xie, L. S.; Wang, C. S.; Zhang, H. Z.; Shu, L.; Bai, J.; Chai, Y. S.; Zhao, X.; Nie, J. C.; Cao, C. B.; Gu, C. Z.; Xiong, C. M.; Sun, Y.; Shi, J.; Salahuddin, S.; Xia, K.; Nan, C. W.; Zhang. J. X. Magnetic domain-wall motion twisted by nanoscale probe-induced spin transfer. Phys. Rev. B 2014, 90, 224407.

12. Boulle, O.; Malinowski, G.; Kläui, M. Current-Induced Domain Wall Motion in Nanoscale Ferromagnetic Elements. Mater. Sci. Eng., R 2011, 72, 159-187.

13. Thiaville, A.; Nakatani, Y.; Miltat, J.; Suzuki, Y. Micromagnetic Understanding of Current-Driven Domain Wall Motion in Patterned Nanowires. Europhys. Lett. 2005, 69, 990996.

14. Thomas, L.; Hayashi, M.; Jiang, X.; Moriya, R.; Rettner, C.; Stuart S. P. Parkin, S.S.P. Oscillatory Dependence of Current-Driven Magnetic Domain Wall Motion on Current Pulse Length. Nature 2006 443, 197-200.

15. Emori, S.; Bauer, U.; Ahn, S.-M.; Martinez, E; Beach, G.S.D. Current-Driven Dynamics of Chiral Ferromagnetic Domain Walls. Nat. Mater. 2013, 12, 611-616. 
16. Miron, I.M.; Moore, T.; Szambolics, H.; Buda-Prejbeanu, L.D.; Auffret, S.; Rodmacq, B.; Pizzini, S.; Vogel, J.; Bonfim, M.; Schuhl, A.; Gaudin, G. Fast Current-Induced DomainWall Motion Controlled by the Rashba Effect. Nat. Mater. 2011, 10, 419-423.

17. Koyama, T.; Chiba, D.; Ueda, K.; Kondou, K.; Tanigawa, H.; Fukami, S.; Suzuki, T.; Ohshima, N.; Ishiwata, N.; Nakatani, Y.; Kobayashi K.; Ono T. Observation of the intrinsic pinning of a magnetic domain wall in a ferromagnetic nanowire. Nat. Mater. 2011, 10, 194-197.

18. Ryu, K.-S.; Thomas, L.; Yang, S.-H.; Parkin, S. Chiral Spin Torque at Magnetic domain Walls. Nat. Nanotechnol. 2013, 8, 527-533.

19. Phung, T.; Pushp, A.; Thomas, L.; Rettner, C.; Yang, S.-H.; Ryu, K.-S.; Baglin, J.; Hughes, B.; Parkin, S. Highly Efficient In-Line Magnetic Domain Wall Injector. Nano Lett. 2015, $15,835-841$.

20. Yang, S.-H.; Ryu, K.-S.; Parkin, S. Domain-Wall Velocities of Up to $750 \mathrm{~m}$ s-1 Driven by exchange-Coupling Torque in Synthetic Antiferromagnets. Nat. Nanotechnol. 2015, 10, 221226.

21. Hertel, R.; Yan, M.; Kakay, A.; Gliga, S. Beating the Walker Limit with Massless Domain Walls in Cylindrical Nanowires. Phys. Rev. Lett. 2010, 104, 057201.

22. Piao, H.-G.; Shim, J.-H.; Djuhana, D.; Kim, D.-H. Intrinsic Pinning Behavior and Propagation Onset of Three-Dimensional Bloch-Point Domain Wall in a Cylindrical Magnetic Nanowire. Appl. Phys. Lett. 2013, 102, 112405. 
23. Lopatin, S.; Ivanov, Yu. P.; Kosel, J.; Chuvilin, A. Multiscale Differential Phase Contrast Analysis With Unitary Detector. Ultramicroscopy 2016, 162, 74-81.

24. Chapman, J. N. T. The Investigation of Magnetic Domain Structures in Thin Foils by Electron Microscopy. J. Phys. D: Appl. Phys. 1984, 17, 623.

25. Donahue, M.; Porter, D. Object Oriented Micromagnetic Framework (OOMMF) The National Institute of Standards and Technology (NIST) Home Page. http://math.nist.gov/oommf/ (accessed Aug 28, 2006).

26. Ivanov, Yu. P.; Vázquez, M; Chubykalo-Fesenko, O. Magnetic Reversal Modes in Cylindrical Nanowires. J. Phys. D: Appl. Phys. 2013, 46, 485001. 
Table of Contents graphic.

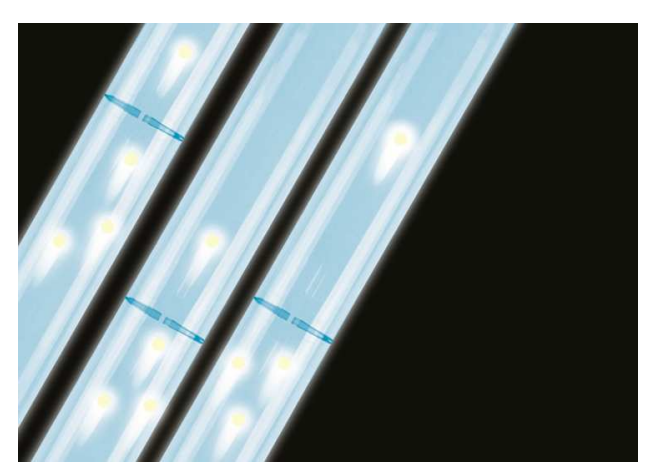

17

18

19

20

21

22

23

24

25

26

27

28

29

30

31

32

33

34

35

36

37

38

39

40

41

42

43

44

45

46

47

48

49

50

51

52

53

54

55

56

57

58

59

60 

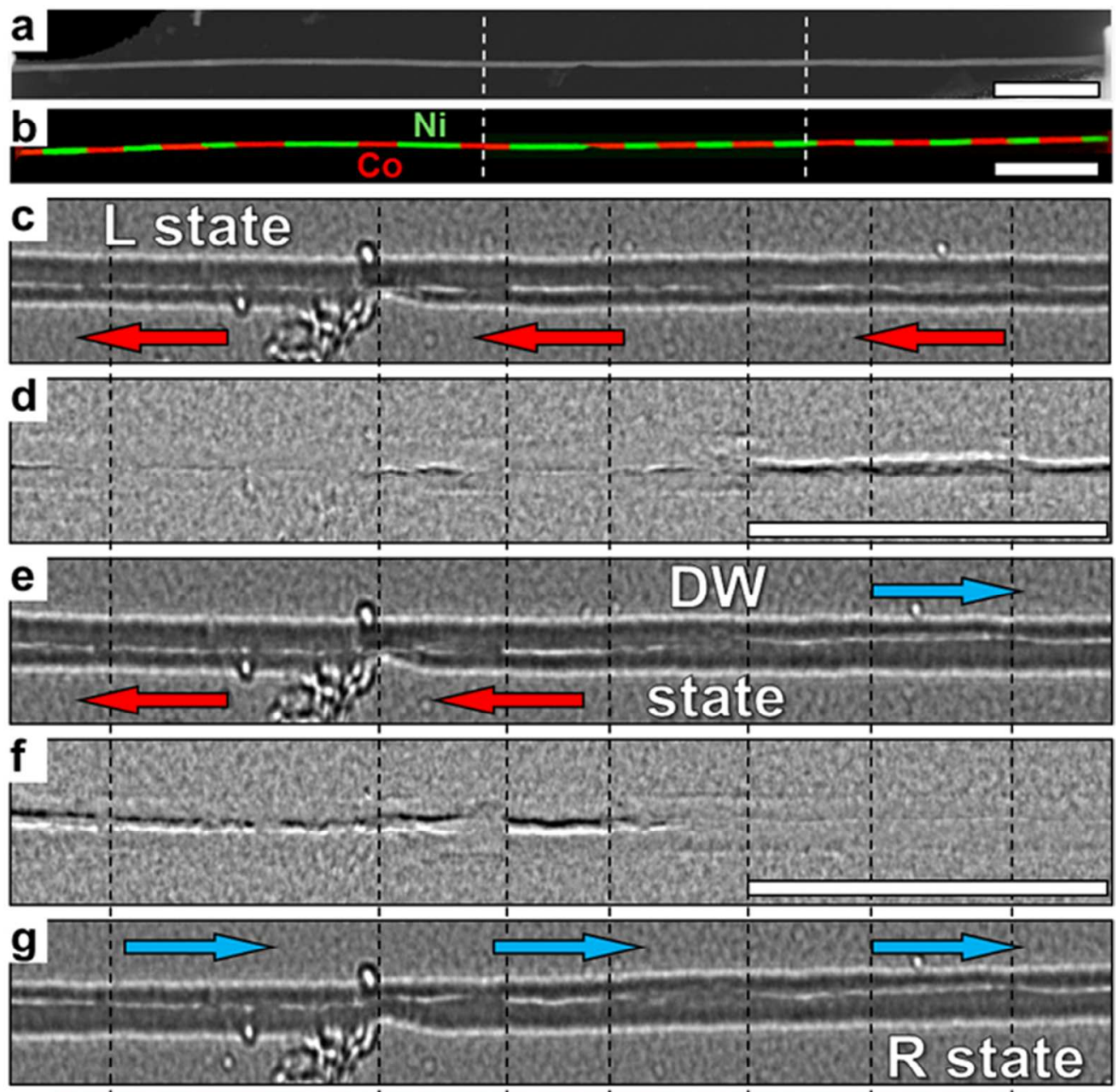

h

(a) STEM image of the device, which consists of a multisegmented Co/Ni nanowire connected to Pt electrodes. (b) EELS elemental mapping of the multisegmented Co/Ni nanowire. (c-g) Overfocused Lorentz images (scaled to $300 \%$ in the vertical direction) of the central section of nanowire designated by white dashed lines in $a$ and $b$ : $c$ and $g$ are the $L$ state and the $R$ state, $e$ is the initial state with the DW pinned at the "interface - DW state," $d$ and $f$ are images of the "DW state - $L$ state," and the "DW state - R state," respectively. (h) EELS elemental mapping of the Co and Ni nanowire segments, the interfaces of which are designated by dashed black lines in c-g. Scale bar is $2 \mu \mathrm{m}$. The complete Lorentz images are in the Supporting Information.

$80 \times 84 \mathrm{~mm}(300 \times 300 \mathrm{DPI})$ 
Diagram representing the magnetic state of the NW shown in Fig. 1 under external magnetic field (B) and electric current.

\section{$115 \times 89 \mathrm{~mm}(300 \times 300 \mathrm{DPI})$}


(a) Domain wall velocity under different current densities Je for various external magnetic field values with respect to the de-pinning field Hdep, simulated at $0 \mathrm{~K}$ temperature. (b) Simulated dependence of the critical current density Jc on the external magnetic field values at $0 \mathrm{~K}$ (circles) and $327 \mathrm{~K}$ (triangles), and experimental data at $327 \mathrm{~K}$ (red " $\mathrm{X}$ ").

$80 \times 32 \mathrm{~mm}(300 \times 300 \mathrm{DPI})$ 


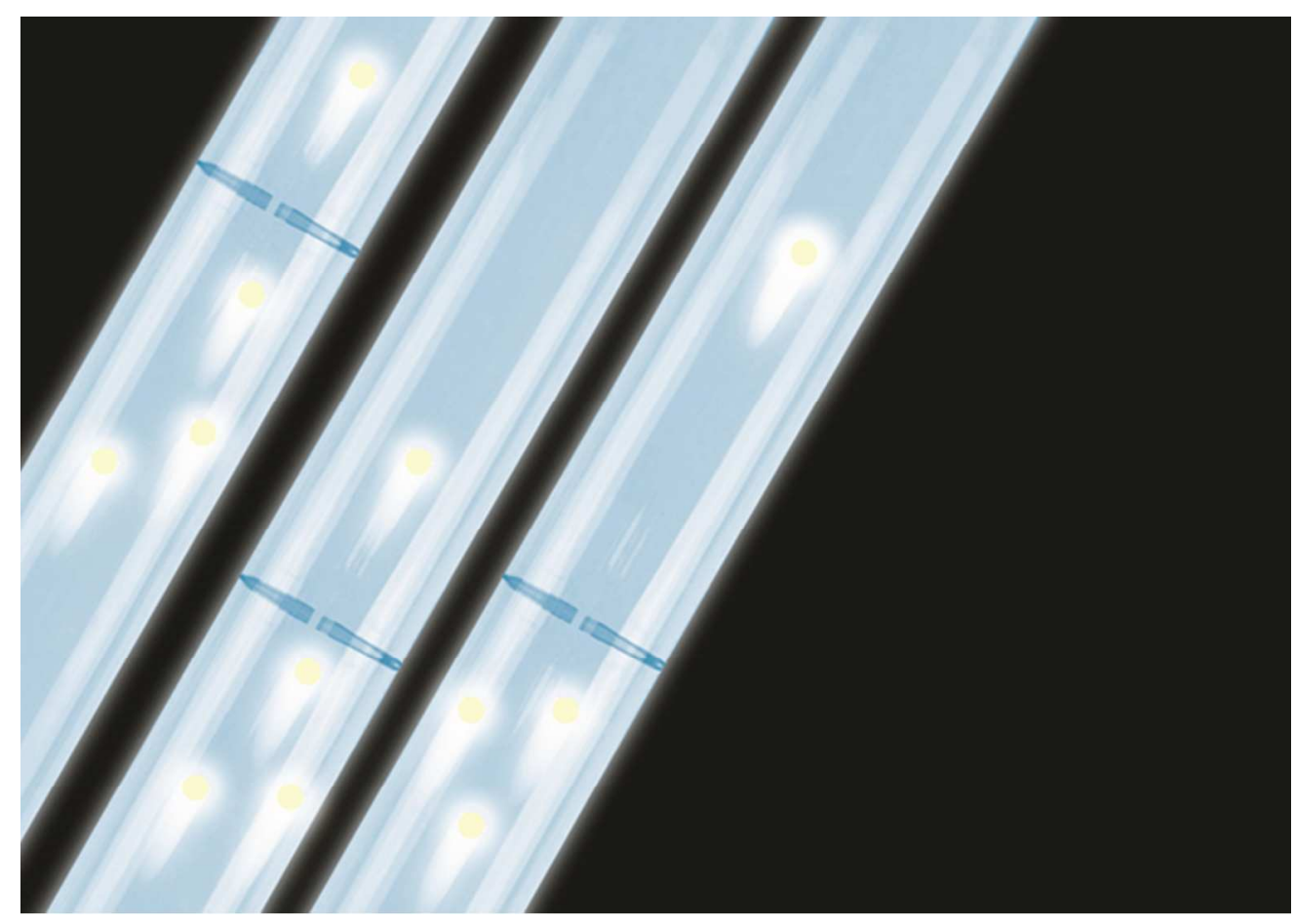

Table of Contents graphic

$62 \times 44 \mathrm{~mm}(300 \times 300$ DPI $)$ 